\title{
Clear aligners for orthodontic treatment?
}

\section{Abstracted from}

Rossini C, Parrini S, Castroflorio T, Deregibus A, Debernardi CL.

Efficacy of clear aligners in controlling orthodontic tooth movement: a systematic review. Angle Orthod 2015; 85: 881-889. doi: 10.2319/061614-436.1. Epub 2014 Nov 20. PubMed PMID: 25412265.

Address for correspondence: Dr Gabriele Rossini, Orthodontic Resident, Department of Orthodontics, Dental School, University of Turin, Via Nizza 230, Turin, Italy. E-mail: dr.gabriele.rossini@gmail.com

\section{Question: Is clear aligner treatment effective in controlling the orthodontic movement in non- growing subjects?}

Data sources PubMed/Medline, Embase, Cochrane Central Register of Controlled Clinical trials (CENTRAL), Web of Knowledge, SCOPUS, Google Scholar and LILACS databases.

Study selection Clinical prospective and retrospective studies of orthodontic treatment with clear aligners on patients over the age of 15 that included clear descriptions of the materials and applied technique were included. Selection was undertaken independently by two reviewers.

Data extraction and synthesis Two reviewers extracted data independently with study quality being assessed using the grading system described by the Swedish Council on Technology Assessment in Health Care (SBU). A narrative summary of the findings was presented. Results Eleven studies involving a total of 480 patients were included consisting of two randomised controlled trials, five prospective studies and four retrospective studies. Six studies were considered to be of moderate quality, the remainder of limited quality.

Conclusions Most of the studies presented with methodological problems: small sample size, bias and confounding variables, lack of method error analysis, blinding in measurements, and deficient or missing statistical methods. The quality level of the studies was not sufficient to draw any evidence-based conclusions.

\section{Commentary}

The demand for orthodontic treatment amongst adults has significantly increased over the last 20 years. There is little doubt that the development of aesthetic appliances such as clear aligners has increased the acceptability of orthodontic care in this group of patients. However, like all forms of treatment, it is imperative that outcomes achieved using such methods are thoroughly explored and supported by high quality research.

The aim of this systematic review was to assess the effectiveness of clear aligner treatment (CAT) in controlling orthodontic tooth movement in non-growing individuals.

A broad search strategy was conducted and the authors took appropriate steps to minimise the potential for selection bias. A total of eleven studies were included; four retrospective non-randomised, five prospective non-randomised and two prospective randomised studies. This provided a total combined sample of 480 patients, and although the authors' inclusion criteria clearly stated that only studies based on human subjects with a minimum age of 15 years would be included, the age range of the combined sample was reported to be 13 to 72 years. Nevertheless, the authors sought to establish the efficacy of CAT in performing intrusion, extrusion, rotation, tipping and alignment movements, all of which are clinically relevant outcomes.

The methodology used was rigorous, and the drawbacks of the review itself are merely a reflection of the limited quality of evidence available in the literature. Of the included studies, six were found to be of moderate quality, whilst the remainder were of limited quality. The review found some evidence to suggest that CAT is effective in producing alignment and levelling of the dental arches in non-growing individuals, and that the degree of anterior intrusion achieved is similar to that attained using the straight wire technique. A retrospective study also found that bodily movement of upper molars is possible when a distilisation of at least $1.5 \mathrm{~mm}$ is prescribed, however there is conflicting evidence regarding the degree of crown versus bodily movement that is achievable using CAT. The review also identified some evidence to suggest that CAT is not effective in controlling anterior extrusive movement or rotations.

Overall, the review suggested that CAT is effective in the management of simple malocclusions. However, the authors acknowledged that the conclusions are strongly limited by the quality of evidence available, and of particular concern was the small sample sizes used, bias and confounding factors present, lack of blinding in outcome assessment and inappropriate use or reporting of statistical methods. The authors recommended that future research assessing CAT should be based on high quality randomised controlled trials, if appropriate recommendations for their use are to be made. Of course, it is crucial that the impact of CAT on patient-reported outcome measures is also assessed, as this will no doubt improve patient compliance and satisfaction.

\section{Practice points}

- Based on the available evidence, it would appear that CAT is effective in the management of simple malocclusions.

- Further high quality research, in the form of randomised controlled trials, is required to confirm such findings, and to explore the impact of such treatment on patient-reported outcomes.

Hanieh Javidi and Elizabeth Graham

School of Clinical Dentistry, Claremont Crescent, Sheffield, UK Evidence-Based Dentistry (2015) 16, 111. doi:10.1038/sj.ebd.6401133 